\title{
Padre Costantino Ruggeri, cantor de la belleza
}

\author{
Father Costantino Ruggeri, the singer of beauty
}

Luigi Leoni

https://doi.org/10.17979/aarc.2009.2.1.5042

\section{INTRODUCCIÓN}

El padre Costantino Ruggeri nació en Adro - localidad italiana próxima al lago de Iseo, en la provincia de Brescia - en 1925, y terminó su laboriosa e intensa vida terrena en 2007. Religioso y artista, supo compatibilizar ambas vocaciones; como él mismo decía, se le había concedido la gracia y la dicha de haber identificado su fe en el arte y su arte en la fe'.

Un hermano suyo en religión, el padre Michele Piccirillo, famoso arqueólogo del Studium Biblicum de Jerusalén, ha escrito: «En las preces de Vísperas de la Liturgia de las Horas, los martes de la tercera semana, la Iglesia nos invita a rezar así: Señor, te rogamos por los artistas a los que has confiado la misión de revelar el esplendor de tu Rostro; haz que sus obras lleven a la humanidad un mensaje de paz y esperanza».

$\mathrm{Y}$ añade: «En ese espíritu creo que deberíamos de agradecer al Señor por todo lo que el padre Costantino ha hecho de bueno y hermoso por la Iglesia y por la Orden Franciscana. Ha sido un artista cristiano y franciscano, con el sagrado fuego de la belleza en su interior. Un cristiano, un sacerdote, vive para embellecer la Iglesia de Dios, viviendo su vida tras la estela de Cristo (...) El padre Costantino ha hecho a la Iglesia más hermosa, viviendo su vida de cristiano y de sacerdote, poniendo a disposición sus dotes de artista, de las que Dios lo ha hecho dueño en abundancia $»^{2}$.
Recorramos brevemente las etapas de su vida.

\section{LA FORMACIÓN TEOLÓGICA Y ARTÍSTICA}

A los doce años entra en el convento, y mientras se prepara para ser fraile franciscano se dedica al estudio del diseño y de la pintura. Luego, durante su época de estudiante de teología en el convento de Busto Arsizio (Varese), experimenta con la técnica de la pintura al fresco, dedicándose al estudio de Masaccio, Piero della Francesca, Giotto y Fra Angélico (Fig. 1-3).

En 1951, recibe su ordenación sacerdotal en el Duomo de Milán. Ese mismo año, Mario Sironi, presentando su primera exposición de pintura en el Centro Culturale San Fedele de la ciudad lombarda, afirma:

«En el duro lenguaje castrense se suele decir quemar etapas. El padre Costantino, que no llega a los veinticinco años, es ya un verdadero soldado de dos ejércitos: el de la fe y el del arte. No pertenece - como ha sucedido casi siempre hasta ahora entre los religiosos- a la categoría de los nostálgicos de la pintura antigua. El padre Costantino plantea hoy ante nosotros que es posible ser creador, revelador e inventor sin estar adscrito al monopolio oficial de la modernidad (...) Sed bienvenido, padre Costantino, al mundo del arte. Este te acoge como a un buen hermano. Deberás tener valentía (...) Tu alma es viva y radiante».

Y proféticamente concluye: «Traes a la Iglesia una 

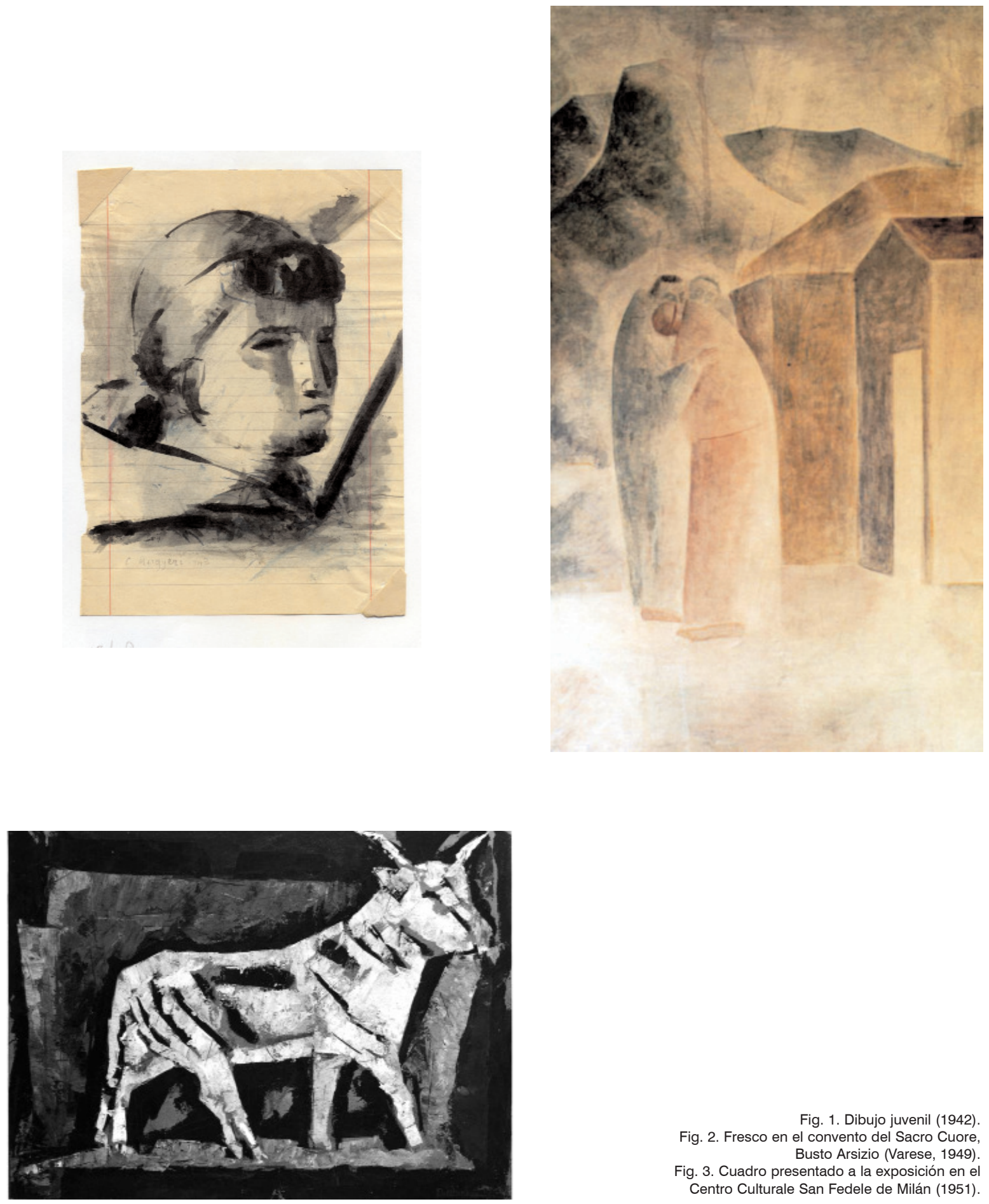

Fig. 1. Dibujo juvenil (1942). Fig. 2. Fresco en el convento del Sacro Cuore, Busto Arsizio (Varese, 1949). Fig. 3. Cuadro presentado a la exposición en el Centro Culturale San Fedele de Milán (1951). 


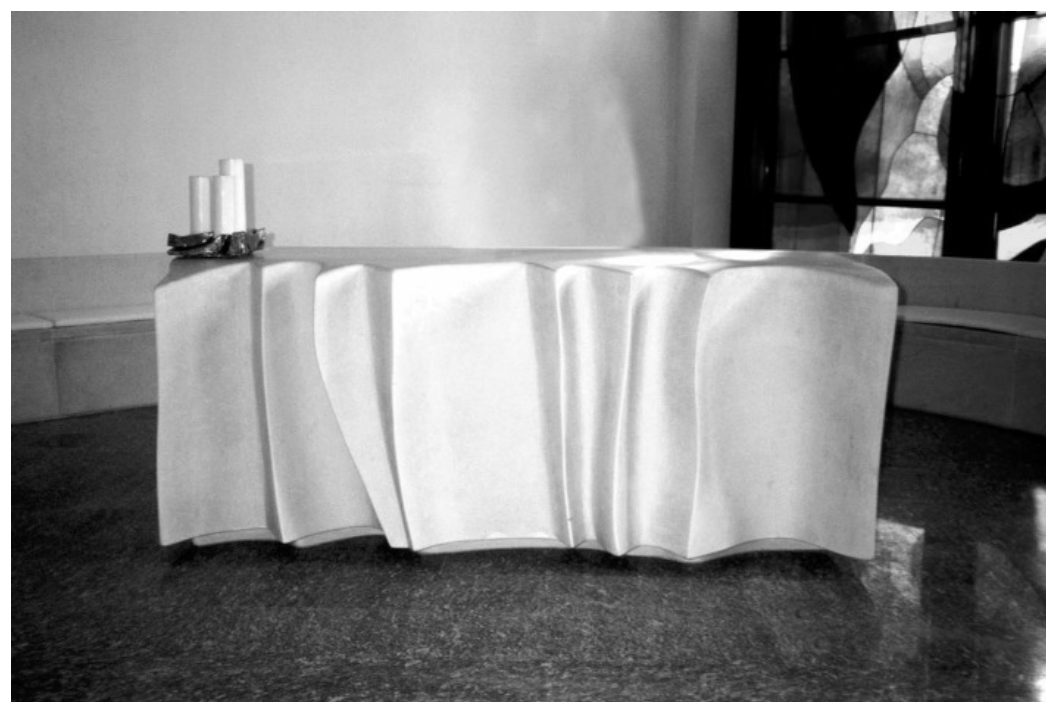

Fig. 5. (En la página siguiente) Padre Costantino Ruggeri con Luigi Leoni (a la derecha) en el convento franciscano de Canepanova, Pavia (1965).

Fig. 4. Altar de la capilla de los PP. Salesianos, Turín (h. 1960).

nueva inteligencia, de la cual ella se beneficiará para desarrollar una evolución que necesita; una evolución de la cual - finalmente y con nostalgia - siente necesidad $»^{3}$.

En Milán prosigue su actividad pictórica, formándose en la escuela de los más grandes pintores, escultores y arquitectos de la posguerra. Conoce y trata a artistas de vanguardia como Fontana, Crippa, Dangelo, Capogrossi, Dova o Meloni, y a los arquitectos Figini, Pollini y Gardella. Atento y abierto a las tendencias artísticas del siglo en curso, se dedica a la experimentación de nuevas poéticas, con lenguajes que huyen de lo tradicional. En pintura, pasa del estilo figurativo a varias experiencias de lo abstracto, haciendo propias las visiones de la contemporaneidad. En 1954 recibe el primer premio San Fedele y el tercer premio Marzotto.

En 1956 entra a formar parte del grupo patrocinado por el Cardenal Lercaro en Bolonia - junto con los arquitectos Glauco y Giuliano Gresleri y Giorgio Trebbi- para el estudio e información sobre arquitectura sacra, y tiene ocasión de reunirse con Le Corbusier y Alvar Aalto.

Desde 1958 a 1962 asiste a la Academia de Brera (Milán), abriéndose a la experiencia escultórica, estudiando con pasión la expresividad de los materiales, trabajando el yeso, la madera, la creta y la piedra (Fig. 4).

Cuando en 1959 se traslada al convento de
Canepanova, en Pavia, donde yo lo conocí (Fig. 5), el padre Costantino se dedica a la realización de todo tipo de objetos de ornamentación sacra ${ }^{4}$. De modo artesanal, realiza pilas de agua bendita, candelabros, cruces, cálices, píxides, sagrarios y custodias (Fig. 6-9).

Se puede hablar del padre Costantino como de un verdadero intérprete de la reforma conciliar en el campo del arte. A fines de los años sesenta se aventura en el campo de los vitrales: para el padre Costantino y para mí, que estaba a su lado, ésta fue una afortunada llamada a vivir de la luz y en la luz, signo admirable del esplendor de Dios.

Después de una experiencia inicial con vidrieras realizadas en paneles de hormigón armado y vidrio tintado procedente de Murano (Venecia), a partir de 1966 comienza a realizar vidrieras de vidrio soplado al estilo antiguo y montado en plomo, destinadas a numerosísimas iglesias y capillas en toda Italia y en el extranjero (Fig. 10). De los años setenta son los Trasparenti, obras consideradas más pictóricas que de escultura, compuestas con vidrios reciclados y hechos revivir con las señales y las huellas del tiempo. Constituyen una de las páginas más líricas y originales de la búsqueda formal del padre Costantino (Fig. 11).

En marzo de 1978 escribe el padre Ruggeri: «El espacio místico constituye el motivo y el fermento de mi experiencia actual. Contrariamente al espacio sacro 


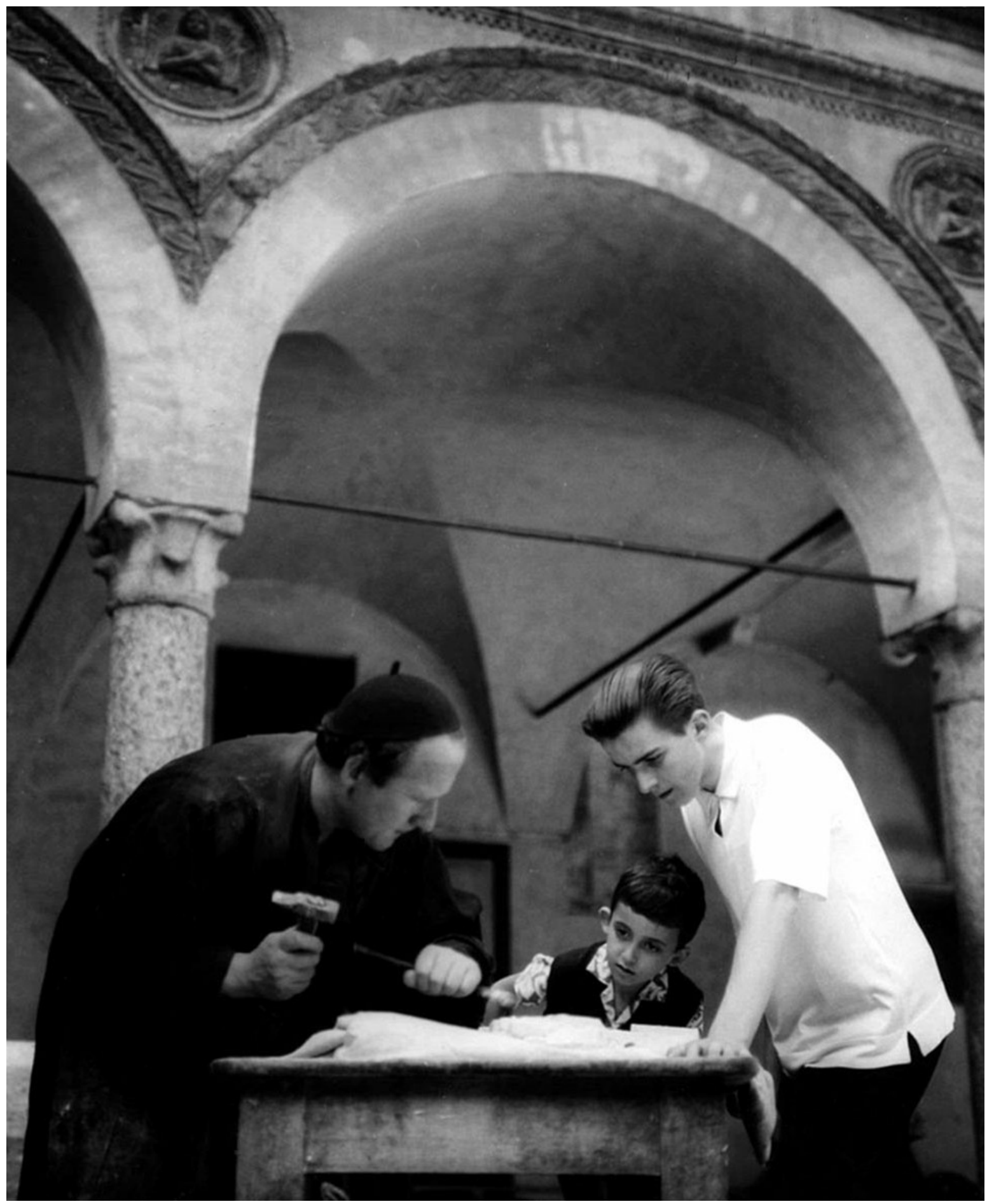



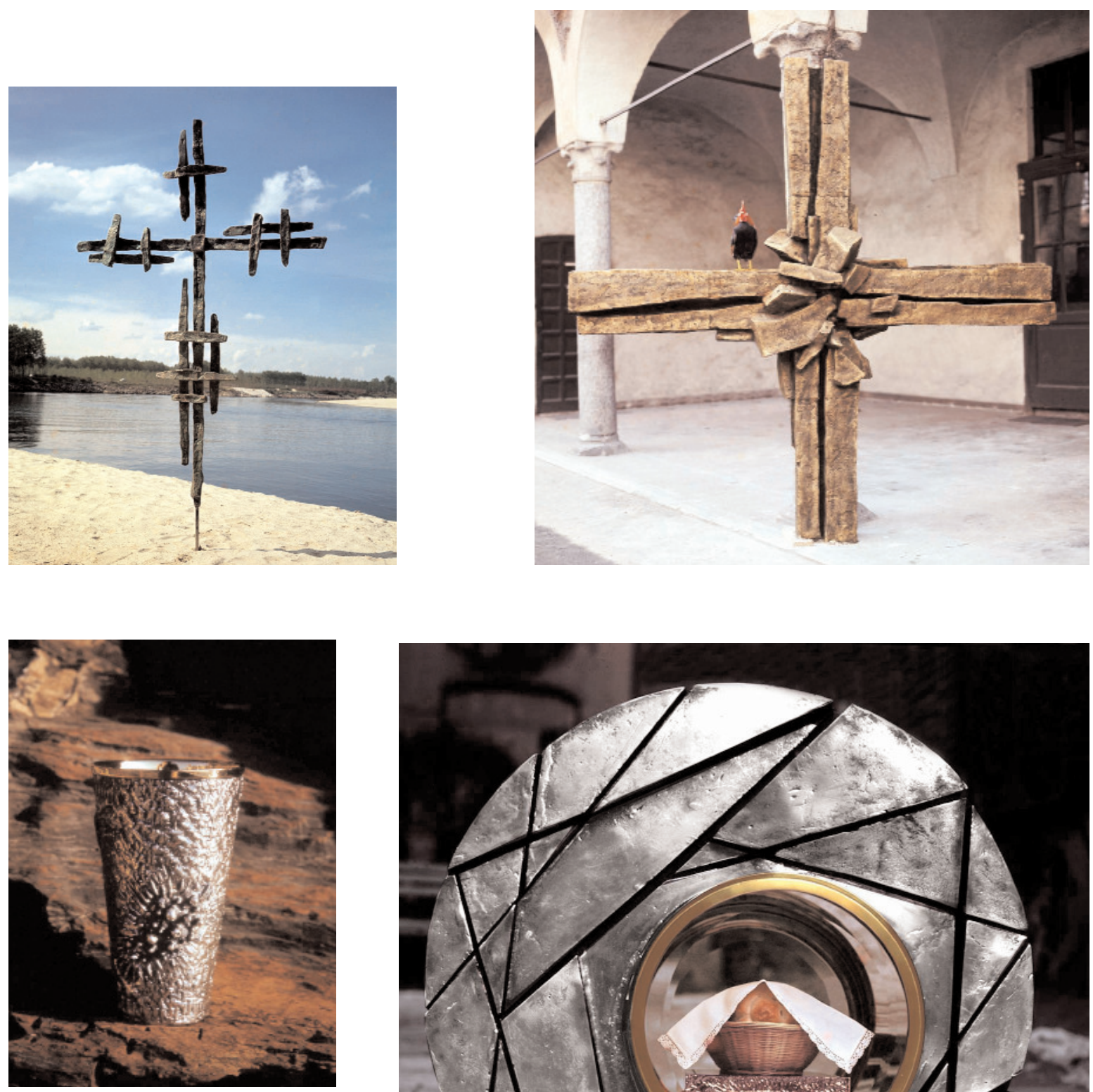

Fig. 6. Cruz gloriosa (h. 1970); bronce.

Fig. 7. Cruz gloriosa (1975); modelo en yeso para realizar en madera.

Fig. 8. Cáliz (h. 1970)

Fig. 9. Sagrario (1969); bronce plateado.

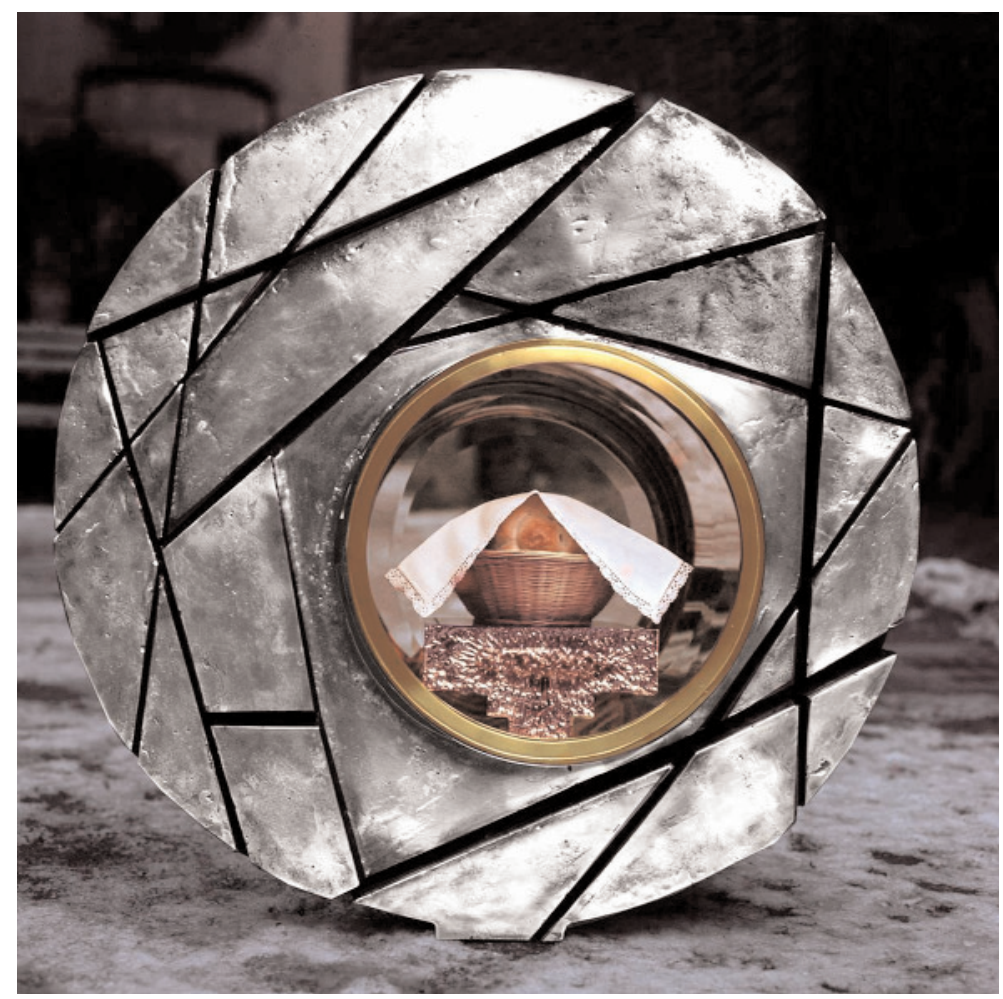


tradicional —que es siempre un espacio circunscrito, definido, configurable en términos volumétricos y estéticos ya asimilados - el místico tiene dimensiones siempre intuidas y nunca definidas. Precisamente por dichas razones, el espacio místico es estimulante. Es, para entendernos, un espacio absoluto y no relativo, un lugar abierto, no cerrado. Puede ser un rayo de sol sobre un muro, una cuerda tensada entre dos mástiles, una flor, una piedra. Buscar la evidencia, para un artista, para un arquitecto, para un pintor o escultor, es la aventura estética más apasionante y difícil, porque es por encima de todo una aventura del hombre, y para mí, también de búsqueda de la sombra de Dios en la luz de lo evidente, o de la luz de Dios en la sombra de las limitaciones de lo creado. El espacio místico es un fragmento, un instante, un aspecto de la belleza y de la verdad de las cosas, una zona libre de instrumentalidad, de funcionalidad, del uso de la sacralización y de la laicización según la moda. Es el punto de encuentro entre lo absoluto de la belleza y lo relativo de la forma en la que se presenta, más que algo terminado y delimitado, continuamente señalado como un punto de partida siempre nuevo para la experiencia del artista ${ }^{5}$.

\section{PRIMERAS OBRAS DE ARQUITECTURA}

De 1982 es la publicación de las Celle (celdas), claro y potente manifiesto del credo artístico del padre Costantino Ruggeri. En la introducción, comienza su discurso de un modo vibrante y encendido:

«Nuestro espacio ya es inhabitable. Todo es oscuro, desproporcionado y modesto. Y sobre todo, insignificante. Las agobiantes aglomeraciones urbanas, las angustiosas calles, las iglesias sin poesía, las desgastadas y corruptas instituciones, los intelectuales sin creatividad, los corazones sin latido; y en todo ello, la renuncia a lo sublime. Ofendidos por este atentado contra las incontenibles esperanzas, buscamos en la completa desnudez los signos humildes y absolutos de un espacio inocente, expresivo para el alma y hospitalario para el corazón. Rechazando toda banalidad y concesión utilitaria al espacio, queremos vivir la más incorruptible y embriagadora poesía de las cosas. Más allá de cualquier intención estética, la propuesta de las Celle —entendida como el núcleo antiguo del más claro y audaz futuro- se dirige hacia el redescubrimiento del primer Edén, del albor del mundo. Un espacio místico más que sacro, no orgánico, sino de imagen, luz y poe- sía; provocador y exaltador de la liberación de las cosas muertas, para despertarse en el fulgor de la belleza, en el blanco luminoso y ardiente» ${ }^{6}$.

Las Celle se construyeron con materiales pobres y de uso cotidiano: cartón, vidrio, madera, yeso, colores de pintura al temple, alambres. Son obras plásticas que revelan el esfuerzo para testimoniar la autonomía de una búsqueda original, fruto de las experiencias adquiridas a nivel espacial (Fig. 12).

Todas las investigaciones artísticas en cada ámbito del arte sacro llevan al padre Costantino a afrontar los temas de la arquitectura sacra en su totalidad. En los años setenta comienza a idear iglesias nuevas. Es una sucesión de episodios que nos hablan de maravillosas sensaciones experimentadas al mismo tiempo.

De 1974 es la primera iglesia importante, dedicada a la Madonna della Gioia en Varese (Fig. 13). La nave eclesial tiene capacidad para ciento cincuenta personas. Un atrio cubierto amplía el espacio para los fieles gracias al ingenioso recurso técnico de unas puertas de bronce que, en caso de necesidad, desaparecen en un hueco del pavimento. Se pone un particular cuidado en la elección de los materiales constructivos: revoco exterior rústico de textura de grano grueso, enlucido interior enrasado de escayola, falso techo de paneles de yeso dispuestos en varios niveles, pavimentación de la iglesia en pizarra, pavimentación del atrio y de las rampas de acceso a la iglesia en cantos rodados, etc.

De 1978 es la iglesia del Tabernáculo en Génova (Fig. 14). La iglesia se apoya suavemente en una pequeña zona en pendiente, situándose de modo discreto y profundo entre la vegetación e integrándose con armonía en el paisaje. La nave eclesial, en anfiteatro, desciende hacia el altar iluminado por un gran lucernario superior. Sobre la cubierta plana se observan un campo de juegos al aire libre y apartamentos para los sacerdotes que dan a pequeños jardines colgantes.

Hagamos referencia también a las experiencias de los años ochenta en las misiones franciscanas de Burundi. En 1967, durante un viaje alrededor del mundo realizado junto al padre Nazareno Fabbretti, periodista y escritor, con la finalidad de conocer las misiones franciscanas, los pueblos, las tradiciones y el arte de diversos países, el padre Costantino tiene oportunidad de comprobar cómo las iglesias construidas por los misioneros aparecen a menudo desprovistas de toda referencia espontánea a la cultura y las tradiciones loca- 

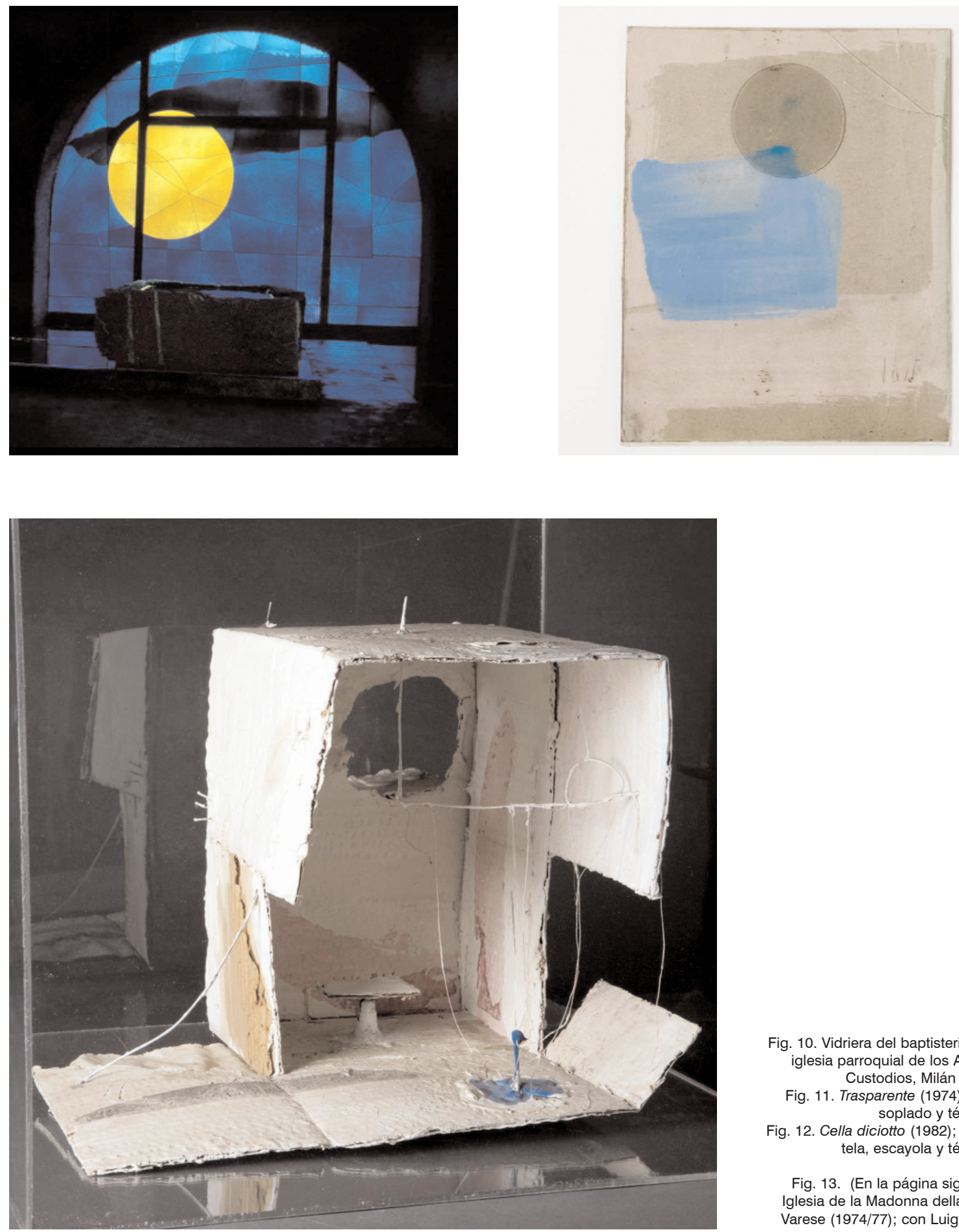

Fig. 10. Vidriera del baptisterio de la iglesia parroquial de los Angeles Custodios, Milán (1971).

Fig. 11. Trasparente (1974); vidrio soplado y témpera.

Fig. 12. Cella diciotto (1982); cartón, tela, escayola y témpera.

Fig. 13. (En la página siguiente) Iglesia de la Madonna della Gioia, Varese (1974/77); con Luigi Leoni. 

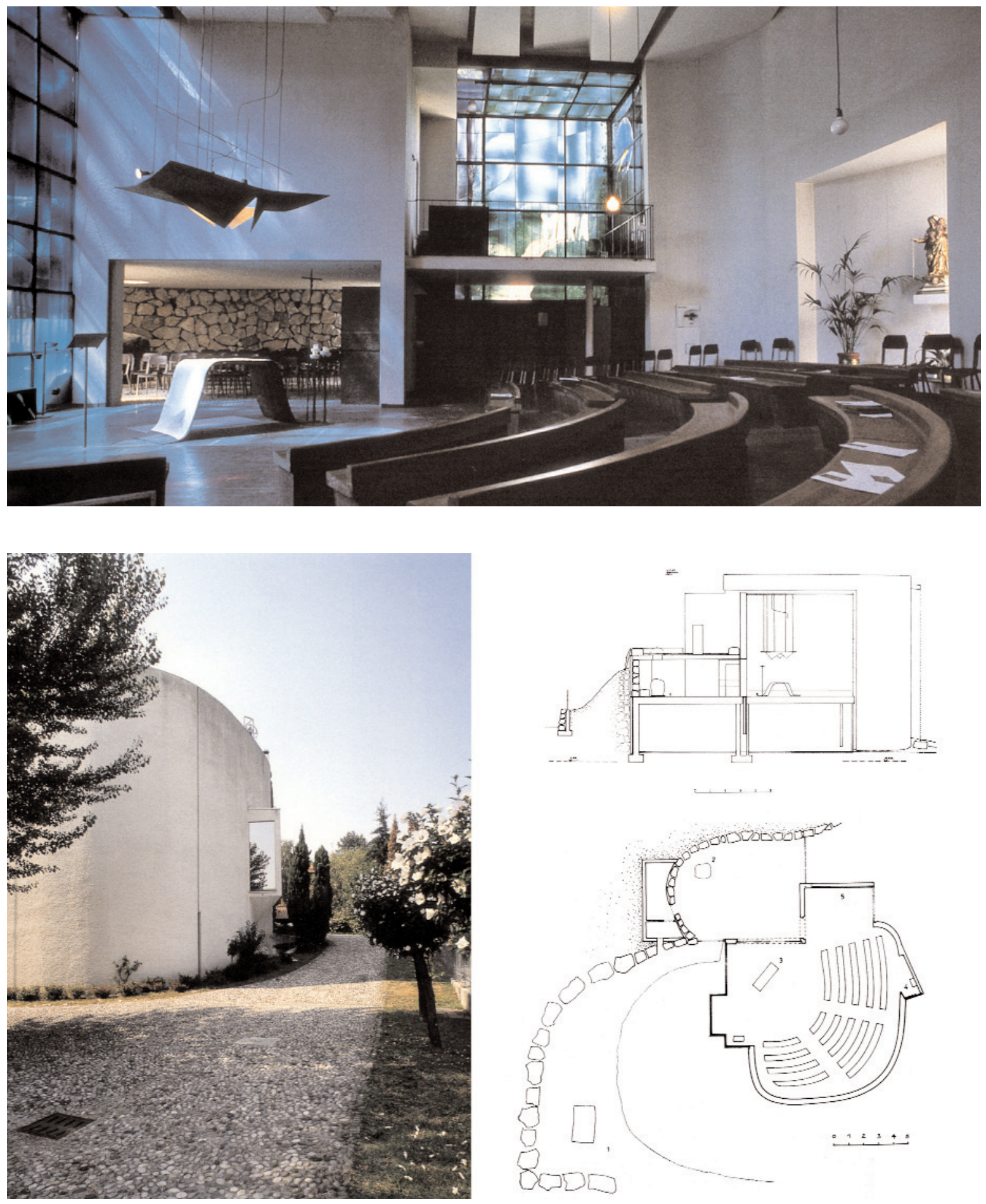


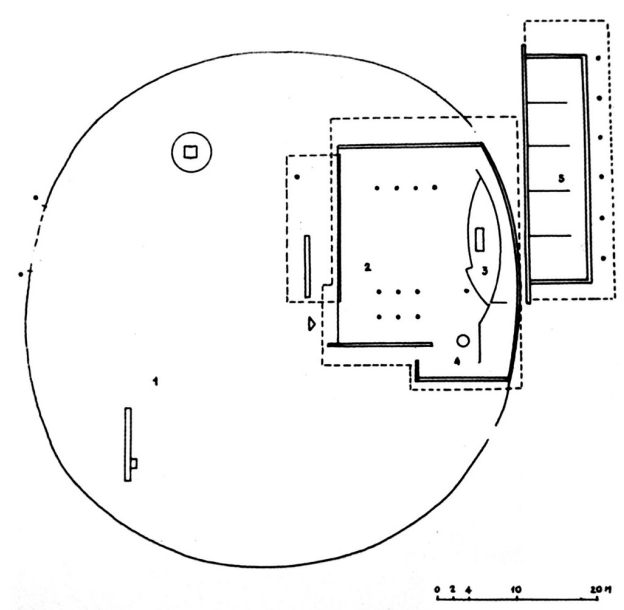

Fig. 15. Iglesia de san Francisco de Asís, Kayongozi (Burundi, 1979/83); con Luigi Leoni.

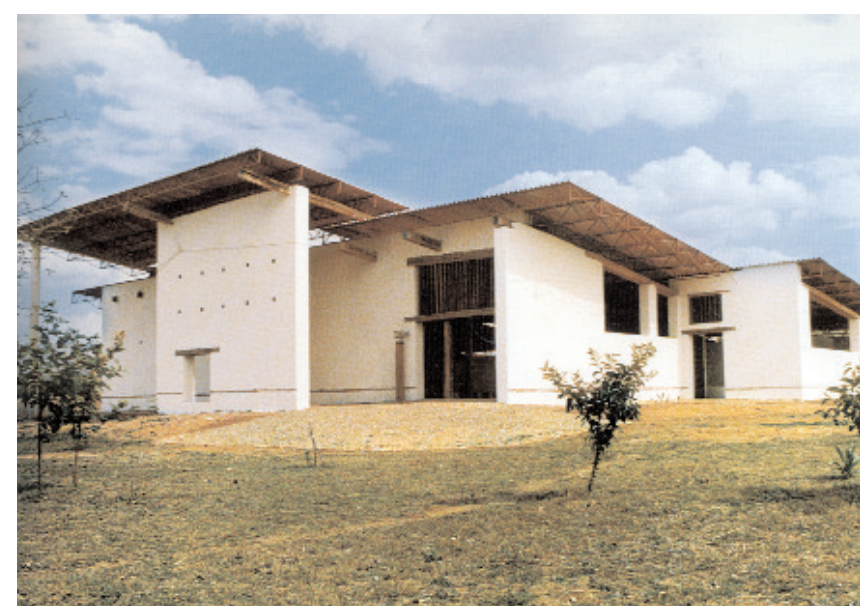

les. Entonces se promete a sí mismo que si se le presenta la ocasión de construir una iglesia en uno de estos países, dicha iglesia tendrá el rostro y el corazón de la tierra y de la gente de la cual nacerá?

La ocasión se presenta doce años después. En su primer viaje a Burundi, realizado en los últimos meses del año 1979, fotografía y dibuja los árboles, los animales, los hombres, sus cabañas, sus útiles domésticos y de trabajo y los materiales de construcción, para sumergirse en la nueva realidad. En Kayongozi, la iglesia se muestra con los materiales constructivos del lugar: madera, piedra, barro y paja, imitando los valores de las cabañas con su rudeza, es decir, con su recinto doméstico. Quiere así emprender un camino que sea una propuesta para profundizar en el problema del respeto a las culturas locales (Fig. 15).

De los años ochenta es el proyecto de la iglesia dedicada a san Bernardo de Claraval, en Roma, incluida en un nuevo y amplio complejo parroquial, con alojamiento para los sacerdotes y diversos edificios parroquiales. Digno de mención es el estudio del área presbiterial con el baptisterio adyacente para la liturgia bautismal por inmersión (Fig. 16).

\section{LA MADONNA DEL DIVINO AMORE, ROMA}

De las más de treinta iglesias proyectadas por el despacho, quiero recordar en concreto el nuevo santua- rio de la Madonna del Divino Amore en Roma, al cual le dedicamos doce años de ferviente trabajo, en un crescendo de realización de promesas y de sueños hasta la celebración del año santo de final de milenio. Ideado en la primavera del año mariano de 1987, fue inaugurado por el papa Juan Pablo II en 1999 (Fig. 17).

El padre Costantino concibe en la fase proyectual al nuevo santuario como un gran macizo de tierra que se eleva y proporciona al espacio inferior la forma de una gruta natural. La estructura portante es de hormigón armado vertido in situ, y la cubierta, de vigas de hormigón prefabricado que descansan sobre un único pilar central diseñado plásticamente. Esta nueva arquitectura es peculiar por su unicidad; tampoco aquí hay referencias a arquitecturas del pasado, ya que una de sus características propias es la novedad, la tensión alrededor de la creatividad poética.

Un segundo aspecto es la preeminencia dada a la manifestación de los valores del espíritu respecto a las expresiones vinculadas al funcionalismo, ya que un espacio sacro - para hablarle al corazón y ponerlo en comunicación con lo trascendente- debe contener en sí la cualidad que lo convierte en una fuente viva de emociones, pero sobre todo de espiritualidad. El espacio está como interiorizado, para invitar a la contemplación y al disfrute místico de los momentos de paz, y en la indiferencia hacia todo lo que es vano y efímero. 

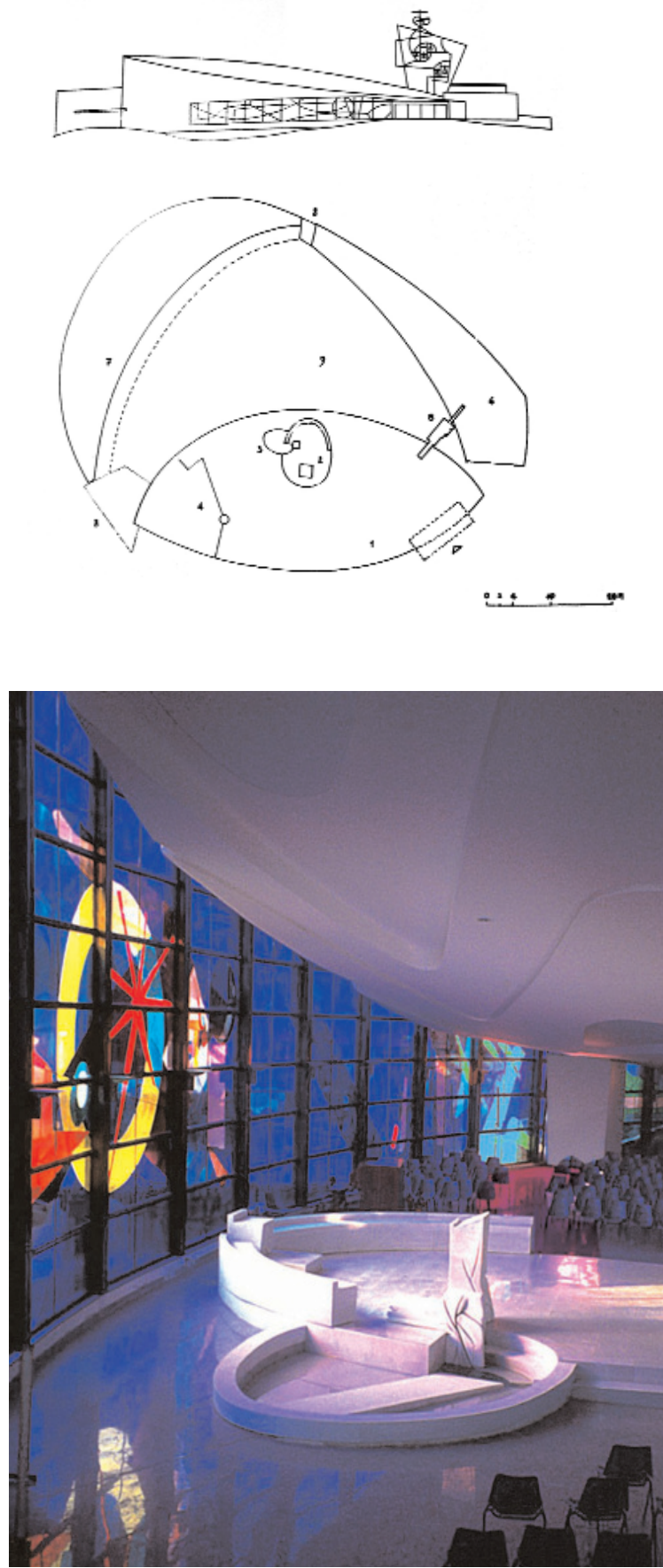

Fig. 16. San Bernardo de Claraval, Centocelle-Roma (1988/93); con Luigi Leoni.
Viene después la búsqueda de una síntesis de pureza e inocencia que manifiesta al máximo la fuerza expresiva y plástica de las formas, búsqueda no desligada del estudio minucioso de la luz, que se introduce como elemento prioritario de significación del misterio que se revela ante nuestros ojos. El hecho de que el haz de luz más potente y más puro se concentre sobre el altar es símbolo de Cristo, y centro de irradiación de los espacios; proviene de las alturas, y es metáfora de la luz de Dios, misteriosa y sublime, que desciende del cielo.

En todo su alrededor, grandes vitrales presentan las innumerables tonalidades del vidrio antiguo soplado y montado con plomo, con la finalidad de dar a toda la nave eclesial una atmósfera altamente espiritual. Se está como inmerso en un espacio inefable, tocado por una Presencia que habla con delicadeza al alma, en un fluir dinámico de instantes siempre cambiantes según la hora del día, las condiciones meteorológicas y las estaciones del año. El organismo espacial resulta así vivo y palpitante, en continua tensión para originar sensaciones e impresiones siempre nuevas.

Desde el punto de vista compositivo, la planta se presenta como una articulación libre de espacios, que llegan a una síntesis formal de aparente sencillez en la envoltura exterior, mientras desarrollan en el interior una variedad de movimientos - sobre todo en torno a la elipse del atrio-, y que genera a espaldas de la gran nave unos recorridos que, a cada paso, se abren a nuevas e inesperadas perspectivas. Aparecen secuencias de líneas rectas con líneas curvas en armonioso contrapunto, para crear una variedad de expresiones no perceptibles a simple vista, sino que necesitan descubrirse dinámicamente, con la apropiación progresiva de los múltiples puntos de vista de los espacios.

La nave tiene una superficie de 1700 metros cuadrados. La capilla eucarística - con una escultura de mármol blanco de Carrara en el centro, en la cual se encaja un sagrario en forma de pan dorado - se asoma al atrio.

Por último, conviene subrayar la relación entre la arquitectura y la naturaleza circundante, lo cual lo convierte en uno de los temas más formidables para tejer sobre él una larga historia de concomitancias y relaciones. Por una parte, la búsqueda de fusión, un intento de esconder entre las ondulaciones naturales de las colinas el gran volumen, de unos 25.000 metros cúbicos, construyendo un prado sobre la cubierta; y por otra, el 

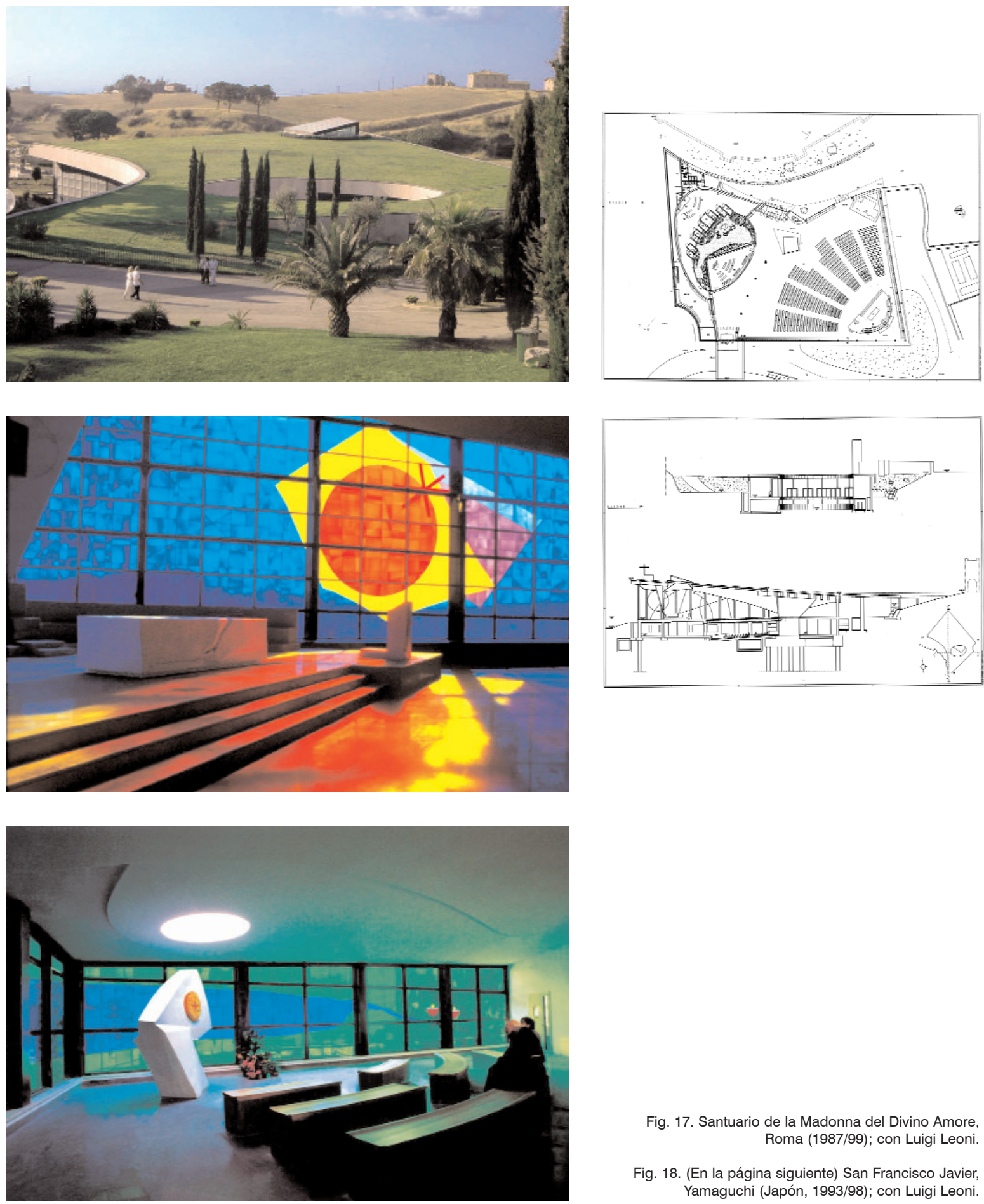

Fig. 17. Santuario de la Madonna del Divino Amore, Roma (1987/99); con Luigi Leoni.

Fig. 18. (En la página siguiente) San Francisco Javier, Yamaguchi (Japón, 1993/98); con Luigi Leoni. 
potente despliegue en el valle de los muros convertidos en inmateriales y diáfanos por la impalpable secuencia de las vidrieras, que nos ofrecen desde el interior un luminoso espejo de la realidad campestre que enmarca al santuario, y que es - ciertamente - uno de los motivos de más atracción para los peregrinos.

\section{UNA OBRA EN JAPÓN}

Contemporáneamente a la ejecución del nuevo santuario del Divino Amore, el padre Costantino es reclamado en Japón para diseñar la iglesia de san Francisco Javier en Yamaguchi, inaugurada en 1998 (Fig. 18). Sencillez de líneas arquitectónicas, esencialidad de los materiales, esplendidez cromática, exaltación de la naturaleza y valoración de los signos litúrgicos, son sus líneas maestras. En un lenguaje actual y diseñando con nuevas técnicas constructivas, se confiere al templo un impulso hacia el cielo para acentuar su sacralidad y la expresividad de los valores trascendentes, convirtiéndolo en un punto focal de atracción y de asombro en la cumbre de una colina que domina la ciudad.

Este planteamiento volumétrico y constructivo hace de esta nueva iglesia un espacio de belleza, de paz y de serenidad, un signo fuerte y sugerente de la presencia católica en Japón para la evangelización de los no creyentes. La fachada del templo tiene como trasfondo dos altas torres, la del reloj a la izquierda y la del campanario a la derecha, que se alzan hacia el cielo y recuerdan en forma actual a las del anterior santuario destruido por un incendio; estos elementos constituyen el símbolo de la ciudad de Yamaguchi.

A través de dos rampas se llega a un amplio atrio que se asoma sobre una plaza verde con un estanque de agua con surtidor. En todo su derredor, una espléndida colina boscosa de iridiscentes colores enfatiza su armónica inserción en la naturaleza. En la amplia fachada resplandece una gran vidriera policromada, que representa el disco solar, símbolo de Cristo, luz y vida del mundo. En la base, un estanque de agua clara quiere ser una llamada a la purificación, antes de acceder a la casa del Señor. Traspasado el atrio cubierto, se llega al pórtico de la iglesia decorado con motivos alegóricos. Desde él se entra en un nartex de noventa metros cuadrados que permite a los visitantes ver el interior de la iglesia a través de un muro de cristal, sin perturbar la celebración de los rituales sagrados. Desde aquí, mediante dos escaleras y un ascensor, también es posi-
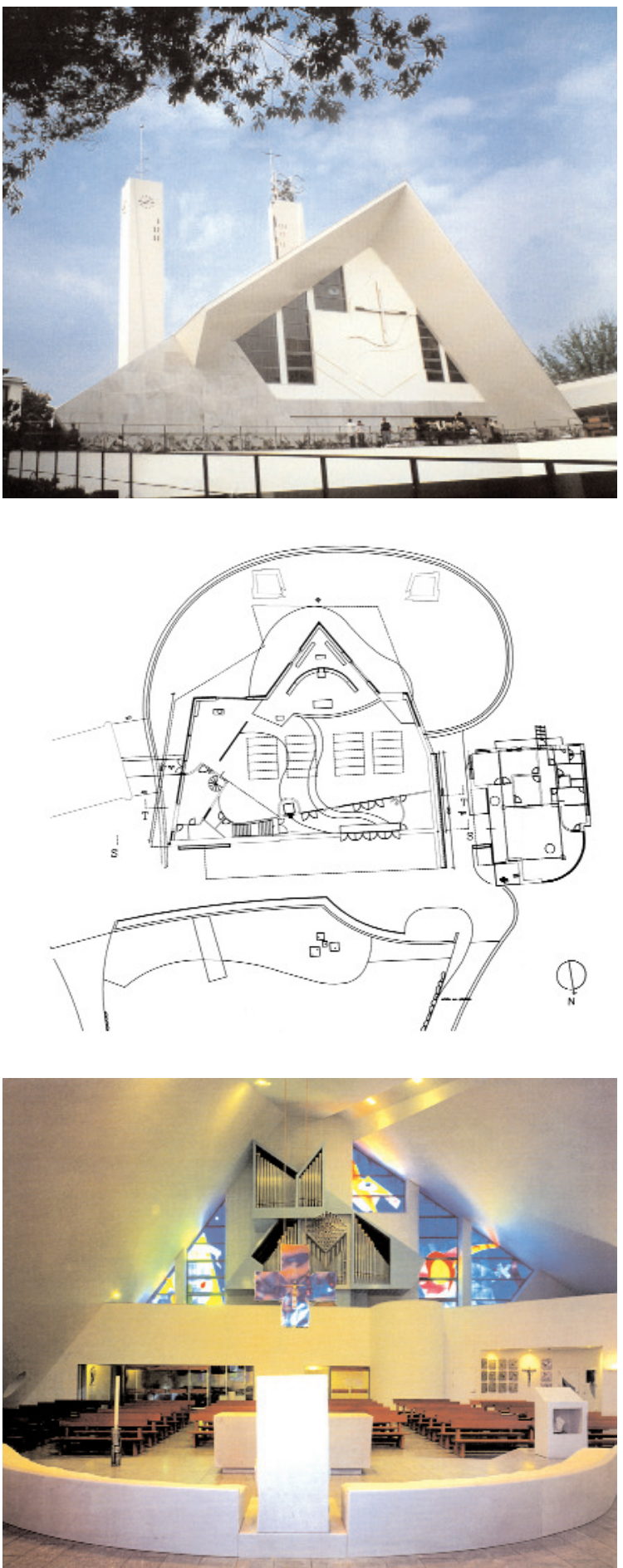


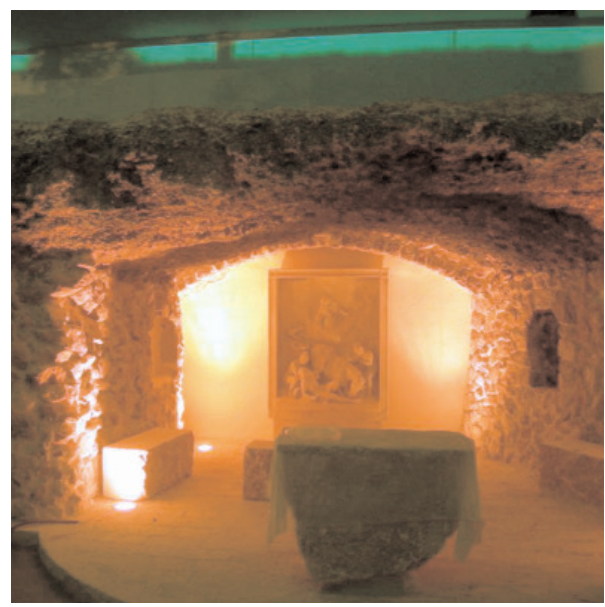

Fig. 19. Cubrición de la gruta de la conversión del apóstol san Pablo en el camino de Damasco (Siria, 2008); con Luigi Leoni y Chiara Rovati.

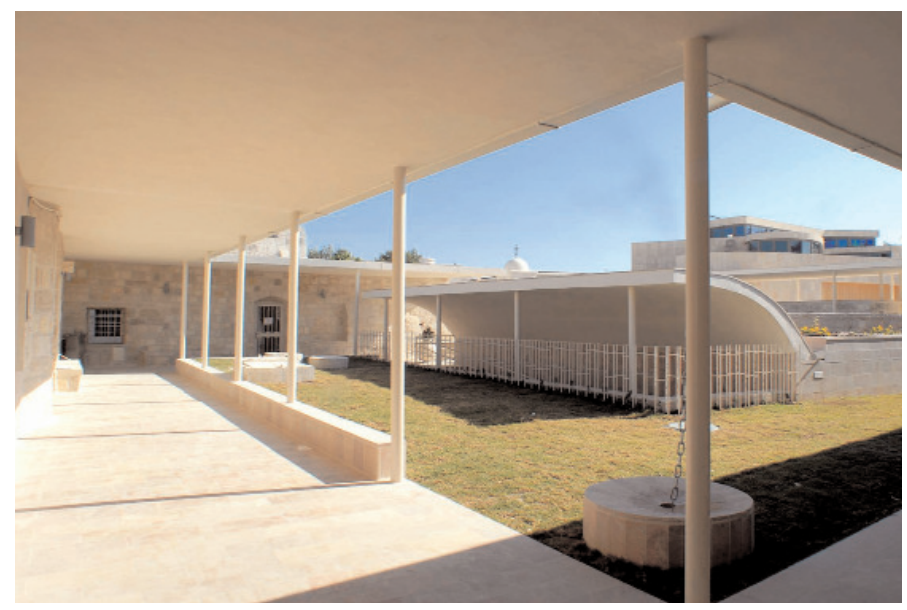

Fig. 20. Santuario de la Madonna della Grotta del Latte, Belén (Israel, 2002/06); con Luigi Leoni y Chiara Rovati. ble comunicarse con los espacios del nivel inferior de la plaza y del nivel superior del matroneo, donde se sitúan el órgano y los cantores. La nave eclesial tiene cuatrocientos metros cuadrados.

El piso desciende ligeramente hacia el presbiterio, convenientemente elevado. En su centro, vuelto hacia el pueblo, se sitúa el altar, símbolo de Cristo: un bloque de mármol blanco, sencillo y bien trabajado, como una flor. Sobre él, en lo alto brilla la cruz de pedrería, símbolo pascual. El baptisterio, según la tradición cristiana hasta el siglo XII, tiene forma de piscina, para subrayar la importancia del misterio de la gracia, de la purificación y del renacimiento que se producen en el alma mediante el sacramento del bautismo.

Los muros perimetrales del templo constan de amplias superficies de vidrieras polícromas montadas en plomo. No solo tienen función decorativa: su finalidad fundamental es la de crear la atmósfera mística de la Jerusalén Celestial, donde aletea perennemente y de modo siempre nuevo la Presencia de Dios, luz cósmica de todas las realidades celestes y terrestres. Los haces de rayos que parten de las vidrieras lanzados por el sol en su recorrido cotidiano, revisten y envuelven no solo el espacio sacro, sino también a los fieles, como realidad y símbolo de la Gracia de Dios que penetra en el alma y llena de alegría los corazones.

La cubierta a varias aguas con diferentes pendientes desciende hasta tocar el suelo, y es soportada por una estructura de cerchas de acero galvanizado ocultas mediante falsos techos de escayola. Desde la plaza de hierba se accede directamente al monumento a san Francisco Javier. Aquí están expuestos cuadros, estatuas y diversos documentos manuscritos y fotográficos que ilustran la historia y el camino de fe realizado por san Francisco Javier y por otros misioneros, en la evangelización de Japón.

\section{6. ÚLTIMOS PROYECTOS}

A comienzos de los años noventa, el padre Costantino Ruggeri pone en marcha la Fondazione Frate Sole, que da testimonio del interés por el estudio y la realización de iglesias que promuevan y exalten los valores del espíritu. Las cuatro primeras ediciones del Premio Internazionale di Architettura Sacra, con periodicidad cuatrienal (1996-2008), han contemplado como ganadores a los arquitectos Tadao Ando, Álvaro Siza, Richard Meier y John Pawson.

En Siria se encuentra la obra para la cubierta de protección de la gruta que guarda la memoria de la conversión del apóstol san Pablo en el camino de Damasco (Fig. 19). Esta obra -inaugurada en 2008 con ocasión de la apertura del Año Paulino- la desarrollé junto con el padre Costantino contando con la colaboración de la arquitecta Chiara Rovati, que ya había participado en 
las realizaciones arquitectónicas de Palestina. Se trata de un mero envoltorio, concebido con materiales naturales, que contiene en su interior la preciada roca. Se emplean bloques de piedra de canteras próximas a Damasco para el altar, el ambón, los asientos del celebrante y de los concelebrantes.

Como culminación de una vida consagrada a la proclamación de la gloria de Dios a través del arte, el padre Ruggeri es reclamado después del año 2000 para la construcción de la nueva capilla de la Theotokos (Madre de Dios), y para la restauración del santuario de la Madonna della Grotta del Latte en Belén, inaugurada en la Navidad del año 2006 (Fig. 20). La nueva iglesia, construida junto a la famosa y antiquísima gruta, es concebida para articular varios espacios en comunica-

Fig. 21. Cristo (1972); trapo sobre tela.

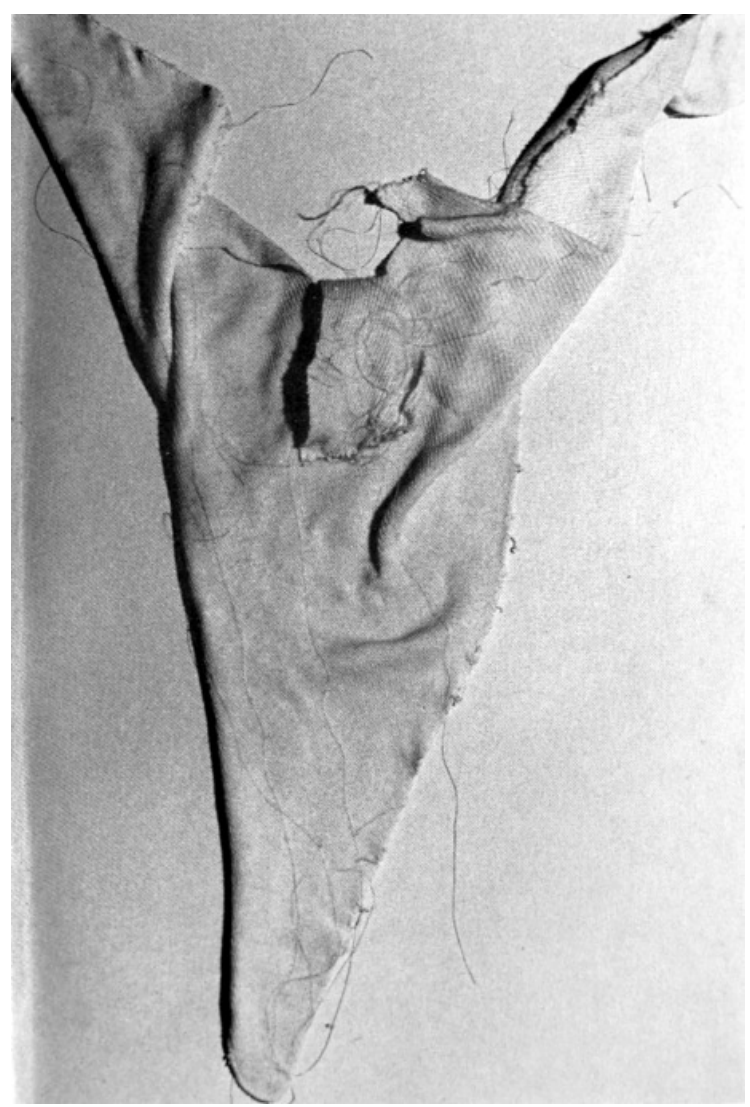

ción con la misma gruta y con el convento de los frailes, ahora habilitado de una forma nueva para albergar un monasterio de monjes de clausura. Sillares abujardados de piedra roja de Belén revisten el edificio principal y todos los muros perimetrales de los corredores del claustro. La planta de la nueva iglesia tiene un diseño libre, espontáneo, es casi una forma generada por la naturaleza. Hallazgos arqueológicos enriquecen los recorridos exteriores.

Aquí, el padre Costantino quiere expresar con fuerza cómo puede un espacio hablarle al corazón del hombre contemporáneo, poniéndolo en comunicación con lo trascendente, a través de la contemplación y el disfrute de instantes de alta espiritualidad (Fig. 21), con el lenguaje de la pureza y de la belleza.

\section{NOTAS}

(1) Nota del editor. Sobre el padre Costantino Ruggeri no existe bibliografía en español. En italiano puede verse, por ejemplo: Antonio Sabatucci (ed.), «Costantino Ruggeri. L'architettura di Dio», Skira, Milán, 2005; Costantino Ruggeri y Nazareno Fabbretti (ed.), «Soltanto un fiore. Genesi di un artista cristiano», Edizioni Dies, Milán, 2001; Costantino Ruggeri y Luigi Leoni, «Spazi di luce 1974-1995», Elle Di Ci Leumann, Turín, 1995; Costantino Ruggeri, «Le celle. Spazi mistici», La Locusta, Vicenza, 1982; Gio Ferri, «Il gesto della spoliazione. Costantino Ruggeri, francescano e architetto», La Locusta, Vicenza, 1980.

(2) Texto escrito por el padre Michele Piccirillo en memoria del padre Costantino Ruggeri, enviado en julio de 2007 y conservado en los archivos de la Fondazione Frate Sole (Pavia, Italia).

(3) Texto incluido en el folleto de presentación.

(4) Nota del editor. Luigi Leoni todavía era un adolescente cuando comenzó a trabajar con el padre Costantino en su taller. Al acabar sus estudios escolares realizó la carrera de arquitectura en Milán, para después asociarse con él. Trabajó con Ruggeri hasta el fallecimiento del franciscano en 2007.

(5) Costantino Ruggeri, «Lo spazio mistico»; Catalogo de la exposición celebrada del 31 de marzo al 23 de abril de 1978 en el Centro Culturale San Fedele de Milán.

(6) Idem., «Le celle. Spazi Mistici», cit.; s/pág.

(7) Cf. Idem. y Luigi Leoni, «Spazi di luce 1974-1995», cit; pág. 66. 